\title{
P14: Der Einfluss von Führungsverhalten und Rollenmodellen auf Händehygienecompliance bei Gesundheits- und Krankenpflegern und Ärzten: Ein narrativer Review
}

\author{
Barbara Kröning • Thomas von Lengerke
}

Online publiziert: 26. Oktober 2013

(C) Springer-Verlag Wien 2013

Fragestellung: Es wird untersucht, ob Führungsverhalten und Rollenmodelle positive Einflüsse auf Händehygienecompliance haben, und ob Unterschiede zwischen Pflegekräften und Ärzten bestehen.

Methodik: In PubMed wurden 21 Studien zum Führungsverhalten und sieben Studien zu Rollenmodellen auf Händehygienecompliance identifiziert.

Ergebnisse: Die Studien, die Rollenmodelle untersuchten, zeigten, dass Compliance und Noncompliance von Kollegen einen Einfluss auf individuelle Händehygiene haben, und einen starken Einfluss von Noncompliance von Führungskräften. Die Studien zum Führungsverhalten zeigten positive Effekte auf die Compliance, wenn Führungsinstrumente die Maßnahmenimplementierung unterstützen. Die Compliance verbesserte sich um 14-56\% auf durchschnittlich 79\%. Elf der eingeschlossenen Studien unterschieden pflegerisches und ärztliches Personal. Hier zeigten sich höhere Complianceraten für Pflegekräfte vor und nach Intervention. Pflegekräfte starteten bei einer durchschnittlich 14\% höheren Compliance. Die Zuwächse beliefen sich auf 6-25\% in der Pflege und $12-33 \%$ bei Ärzten. Zwei Stu- dien benannten Unterschiede zwischen pflegerischem und ärztlichem Personal bei der Auswahl von Rollenmodellen, wobei in der Pflege gute Compliance Rollenmodelle definierte, während bei Ärzten die Erwartung an ein Rollenmodell mit guter Compliance assoziiert war. Eine Studie beschrieb die Krankenhausleitung als für Ärzte wichtigen Faktor, während für die Pflege große Arbeitsbelastungen mit dem Tragen von Kitteln und Handschuhen assoziiert waren. Diskussion: Während die Heterogenität der eingeschlossenen Studien auch bzgl. der Operationalisierung von Händehygiene metaanalytische Auswertungen erschwert, könnte sie auch für studiendesignunabhängige Einflüsse von Führungsverhalten und Rollenmodellen sprechen. Insgesamt legt die große epidemiologische Bedeutung nosokomialer Infektionen die Implementierung von Programmen zur Verbesserung der Händehygienecompliance nahe. Multimodale Interventionen, die von Führungskräften und Rollenmodellen unterstützt werden und berufsgruppenspezifisch angelegt sind, können Händehygienecompliance erfolgreich fördern.

B. Kröning $\cdot$ T. von Lengerke

Medizinische Hochschule Hannover,

Forschungs- und Lehreinheit Medizinische Psychologie,

Hannover, Deutschland 